\title{
Magnetic resonance imaging of Guillain-Barré syndrome
}

\author{
Hong-Liang Zhang • Jian-Meng Wang • \\ Hong-Wei Zhou $\cdot$ Jing $\mathrm{Xu} \cdot$ Jiang $\mathrm{Wu}$
}

Received: 22 June 2010 / Accepted: 7 July 2010 /Published online: 20 July 2010

(C) Springer-Verlag 2010

\section{Dear Editor,}

We read with great interest the article by Yikilmaz and colleagues, which described features of spinal magnetic resonance imaging (MRI) in children with Guillain-Barré syndrome (GBS) and investigated the correlation between MRI characteristics and the clinical/laboratory examinations [2]. Their findings are intriguing in that MRI appears promising as a supplementary modality to conventional diagnostic methods of GBS. However, we have some concerns about this study.

In this study, a control group seems missing, although the authors mentioned "All patients and the control group were scanned with a 1.5-T clinical MR scanner" in the Patients and Methods only. As a consequence, the

H.-L. Zhang $\cdot$ J. Xu $\cdot$ J. Wu

Department of Neurology, First Hospital of Jilin University,

Changchun, China

H.-L. Zhang $(\bowtie)$

Department of Neurobiology, Care Sciences and Society,

Karolinska Institute,

Novum, plan 5,

SE 14186 Stockholm, Sweden

e-mail: Hongliang.Zhang@ki.se

J.-M. Wang

Department of Cadre Ward, First Hospital of Jilin University, Changchun, China

\section{H.-W. Zhou}

Department of Radiology, First Hospital of Jilin University, Changchun, China specificity of contrast enhancement in spinal MRI, which may differentiate GBS from other diseases with/without nerve root involvement, is unavailable. This pitfall of the study design might limit the merits of their findings. Therefore, the conclusion that MRI can be used as a supplementary diagnostic modality to clinical and laboratory findings of GBS should be further confirmed by controlled studies in the future.

Another concern arising from the research is the enrollment of the study subjects. Since the subjects were retrospectively selected from patients who were hospitalized in their center with the diagnosis of GBS between 2005 and 2010, we are eager to know how the authors could accumulate up to 40 GBS cases that underwent a contrast-enhanced spinal MRI. It is well established that MRI contributes to the diagnosis of GBS by demonstrating anterior and posterior intrathechal spinal nerve roots [1]. However, the use of MRI especially contrast-enhanced spinal MRI in GBS is currently uncommon. Considering the relatively low incidence of GBS in children and that this was a single-center study, we suspect that the authors designed and conducted this investigation in a prospective way.

\section{References}

1. Coskun A, Kumandas S, Pac A, Karahan OI, Gulec M, Baykara M (2003) Childhood Guillain-Barré syndrome. MR imaging in diagnosis and follow-up. Acta Radiol 44:230-235

2. Yikilmaz A, Doganay S, Gumus H, Per H, Kumandas S, Coskun A (2010) Magnetic resonance imaging of childhood GuillainBarre syndrome. Childs Nerv Syst. doi:10.1007/s00381-0101197-8 